\title{
Fortgesetzte Untersuchungen über die synthetischen Fähigkeiten der tierischen Zelle. Versuche ubber die Verwertung verschiedener Stickstoffquellen im Organismus des Hundes.
}

\author{
Von
}

Emil Abderhalden und Paul Hirsch.

(Aus dem physiologischen Institute der Universität Halle a. S.)

(Der Redaktion zugegangen am 18. September 1912.)

Wir haben die Versuche über die Verwertung verschiedenartiger Stickstoffquellen im Organismus des Hundes fortgesetzt. ${ }^{1}$ ) Einmal wurden Am mon salze als einzige Stickstoffquelle verabreicht, dann Harnstoff, ferner Gemische verschiedenartiger stickstoff haltiger Substanzen: Acetamid, Benzamid, Guanidin, l-Tyrosin, d-Glutaminsäure, ein Gemisch von Glykokoll und d-Alanin (als Aminosäuregemisch bezeichnet), endlich verschiedene Ammonsalze: Ammonacetat, -phosphat, -valerianat, -lactat, -citrat, -tartrat. Die Versuchsanordnung ist in der folgenden Arbeit (Seite 21 dieses Bandes) genau geschildert, dort findet sich auch eine eingehrende Besprechung der erhaltenen Resultate. Es seien hier deshalb nur diejenigen Fragestellungen hervorgehoben, die in den vorhergehenden Arbeiten noch nicht mitgeteilt sind.

Bei Versuch I versuchten wir einen Teil von in Form von Fleisch verabreichtem Stickstoff durch Ammonacetat zu 'ersetzen, resp. mit dieser Verbindung den Stickstoffstoff wechsel iso zu beeinflussen, daß die Stickstoffbilanz trotz geringerer Stick'stoffzufuhr in Form von Fleisch annähernd gleich blieb. Wir tteilen den Versuch mit, obgleich wir der Meinung sind, daß 'er die gestellte Frage aus verschiedenen Gründen nicht ein(deutig beantwortet. Er ist deshalb für unsere Fragestellung von allgemeiner Bedeutung, weil er zeigt, daß ohne Zweifel

1) Vgl. die Mitteilungen in dieser Zeitschrift, Bd. 78, S. 1; Bd. 80, S. 136 und S. 160, 1912. 
richtige Stickstoffretentionen vorhanden waren, die, sobald ein Hungertag eingeschaltet wurde, zur gesteigerten Stickstoffausfuhr führten. So wurde am 21. Hungertag eine Stickstoffausscheidung von $2,44 \mathrm{~g}$ und am 29. Tage eine solche von $4,71 \mathrm{~g}$ beobachtet. Wir werden die gestellte Frage mit anderer Versuchsanordnung wieder aufnehmen und vom Stickstoffminimum ausgehen.

Versuch II wurde zur Prüfung des Einflusses verschiedener Stickstoffquellen auf die Stickstoffbilanz angestellt. Vor allen Dingen wurde die Wirkung des Harnst offes verfolgt. Grafe ${ }^{1}$ ) gibt in seiner letzten Arbeit an, «daß sich anscheinend auch mit Harnst off ein N-Gleichgewicht erzielen läßt.. Die Belege zu dieser Feststellung sind allerdings noch nicht erschienen. Unsere Versuche ergeben keine Andeutung einer Sparwirkung. Auch mit den übrigen stickstoff haltigen Substanzen konnte die Stickstoffbilanz nicht eindeutig beeinflußt werden. Schien einmal eine Sparwirkung einzutreten, dann folgte bald eine stark erhöhte Stickst offausscheidung. Erwähnt sei, daß manche der verfütterten stickstoffhaltigen Substanzen keineswegs indifferent waren. Es seien einige Arbeiten angeführt, die sich mit der Wirkung der von uns verwendeten Substanzen beschäftigen. ${ }^{2}$ )

1) E. Grafe, Weitere Mitteilungen über die Eiweiß sparende Wirkung verfütterter Ammoniaksalze. Diese Zeitschrift, Bd. 78, S. 500, 1912.

2) 0. Schultzen u. M. Nencki, Die Vorstufen des Harnstoffs im tierischen Organismus. Zeitschrift für Biologie, Bd. 8, S. 124-146 (1872) (Acetamid unverändert im Harn).

W. v. Knieriem, Beiträge zur Kenntnis der Bildung des Harnstoffs im tierischen Organismus. Zeitschrift für Biologie, Bd. 10, S. 263 bis 294 (1874).

E. Salkowski, Über den Vorgang der Harnstoffbildung im Tierkörper und den Einfluß der Ammonsalze auf denselben. Diese Zeitschrift, Bd. 1, S. 1-59 (1877/78).

E. Hallervorden, Über das Verhalten des Ammoniaks im Organismus und seine Beziehung zur Harnstoffbildung. Archiv für exp. Path. u. Pharm., Bd. 10, S. 125/146 (1878).

E. Nebelthau, Über die Wirkungsweise einiger aromatischer Amide und ihre Beeinflussung durch Einführung der Methyl- oder Äthylgruppe. Archiv für exp. Path. u. Pharm., Bd. 36, S. 451-466 (1895).

E. Pommerrenig, Über Guanidinzersetzung im Tierkörper. Beiträge zur chem. Physiologie und Pathologie, Bd. 1, S. 561-566 (1902). 
Auch der Versuch an Hund VII läßt keine Änderung der Stickstoffbilanz nach Eingabe von Harnst off im Sinne einer Retention erkennen; während die Eingabe von Ammonace tat ohne Zweifel eine solche bewirkte.

Der Versuch an Hund VIII zeigt unsere Bestrebungen, der von Grafe angenommenen Eiweißsynthese aus Ammonsalzen und anderen Stickstoffquellen und ferner Kohlenhydraten möglichst günstige Bedingungen zu schaffen. Die Stickstoffzufuhr war eine sehr erhebliche. Es gelang in keiner Weise, die Stickstoffbilanz zu einer positiven zu gestalten. Auch Stickstoffgleichgewicht wurde nicht annähernd erreicht. Die Stickstoffbilanz blieb im Gegenteil stark negativ und an den Hungertagen trat wiederholt eine überraschend große Stickstoffausscheidung ein. Den günstigsten Einfluß hatte ohne Zweifel auch hier das Ammonacetat. Über die Bedeutung des zum Schlusse noch verabreichten Oryzanins unterrichtet die schon erwähnte Arbeit (dieser Band, Seite 52).

Der an Hund IX ausgeführte Versuch demonstriert in sehr klarer Weise, daß es gelingt, das Körpergewicht des Hundes ohne jede Stickstoffzufuhr längere Zeit annähernd im Gleichgewicht zu erhalten, wenn genügend Kalorien zugeführt werden. Das Versuchstier verlor während einer 21 Tage umfassenden, ganz stickstofffreien Periode nur $300 \mathrm{~g}$, nachdem es 10 Tage

J. H. Führer, Die periphere Wirkung des Guanidins. Archiv für exp. Path. u. Pharm., Bd. 58, S. 1-49 (1908).

C. Voit, Untersuchungen über die Ausscheidungswege der stickstoffhaltigen Zersetzungsprodukte aus dem tierischen Organismus. Zeitschrift für Biologie, Bd. 2, S. 6-77, 187-243 (1866).

E. Heilner, Über die steigernde Wirkung des subcutan eingeführten Harnstoffes auf den Eiweißstoffwechsel. Zeitschrift zur Biologie, Bd. 52, S. 216/235, 1910.

Hermann Fühner, Über den Angriffsort der peripheren Guanidinwirkung. Archiv für experim. Path. u. Pharm., Bd. 65, S. 401, 1911.

N. Janney, Die Ammoniakausscheidung im menschlichen Harn bei Zufuhr von Harnstoff und Natron. Diese Zeitschrift, Bd. 76, S. 99 bis $135(1911 / 12)$.

1) E. Grafe, Zur Frage der Eiweißsynthese bei Fütterung von Ammoniaksalzen. Verhandl. des Deutschen Kongresses für innere Medizin. S. 507, 1912. 
lang sein Gewicht nicht nur beibehalten, sondern sogar vermehrt hatte. Die Ammonacetatperiode zeigt eine gewisse Stickstoffretention. Der Versuch, Fleischstickstoff durch Ammonacetat zu ersetzen, führte zu einem negativen Ergebnis.

Was die stickstofffreien Perioden anbelangt, so sei ausdrücklich hervorgehoben, daß die verabreichten Nahrungsstoffe stets auf Stickstoff untersucht wurden. Die Kohlenhydrate waren stickstofffrei. Der Speck enthielt ab und zu bis zu 0,2\% Stickstoff. Selbstverständlich wurde nur ganz undurchwachsener Speck angewandt und zu den stickstofffreien Perioden nur solcher verwandt, der einen Stickstoffgehalt bei der Analyse nicht ergab. Es bleibt bei solchen Bestimmungen nichts anderes übrig, als viele Proben zu untersuchen. Die Verbrennung großer Massen von Fett mit Kjeldahl-Schwefelsäure ist sehr schwierig. Unsere Angaben, daß das verfütterte Fett stickstofffrei war, besagen, daß jedenfalls der Gehalt an Stickstoff so gering war, daß wir mit der Kjeldahl-Methode ihn nicht nachweisen konnten. Wurde Fett verfüttert, das Stickstoff enthielt, dann ist dieser Stickstoffgehalt selbstverständlich in Rechnung gesetzt.

Der Speck enthält nun häufig Salpeter. Diese Art der Stickstoffbindung würde bei der Feststellung des Stickstoffgehaltes nach Kjeldahl dem Nachweis entgehen. Wir wählten deshalb, um ganz sicher zu gehen, Speck, der frei von Salpeter war. Diese Vorsichtsmaßregel erwies sich als wichtig, weil Grafe folgende Mitteilung macht: «Erwähnt sei nur noch zum Schluß, daß mit Salpeter (Natriumnitrat) angestellte Versuche in ziemlich analoger Weise ausfallen, so daß also auch in dieser Richtung der Unterschied zwischen Tier- und Pflanzenreich sich sehr stark verwischen dürfte.» Die Mitteilung der Belege zu dieser Behauptung stehen noch aus. Eigene Versuche ergaben bis jetzt eine vollständig unveränderte, quantitative Ausscheidung des zugeführten Salpeterstickstoffs. Wir werden über diese Versuche demnächst ausführlich berichten.

Der eine von uns (A.) hat ferner gemeinsam mit Ginsberg Versuche begonnen, die feststellen sollten, ob einzelne Aminosäuren imstande sind, als einzige Stickstoffquelle bei reichlicher Zufuhr von Kohlenhydraten und Fett die Stickstoff- 
Über die synthetischen Fähigkeiten der tierischen Zelle.

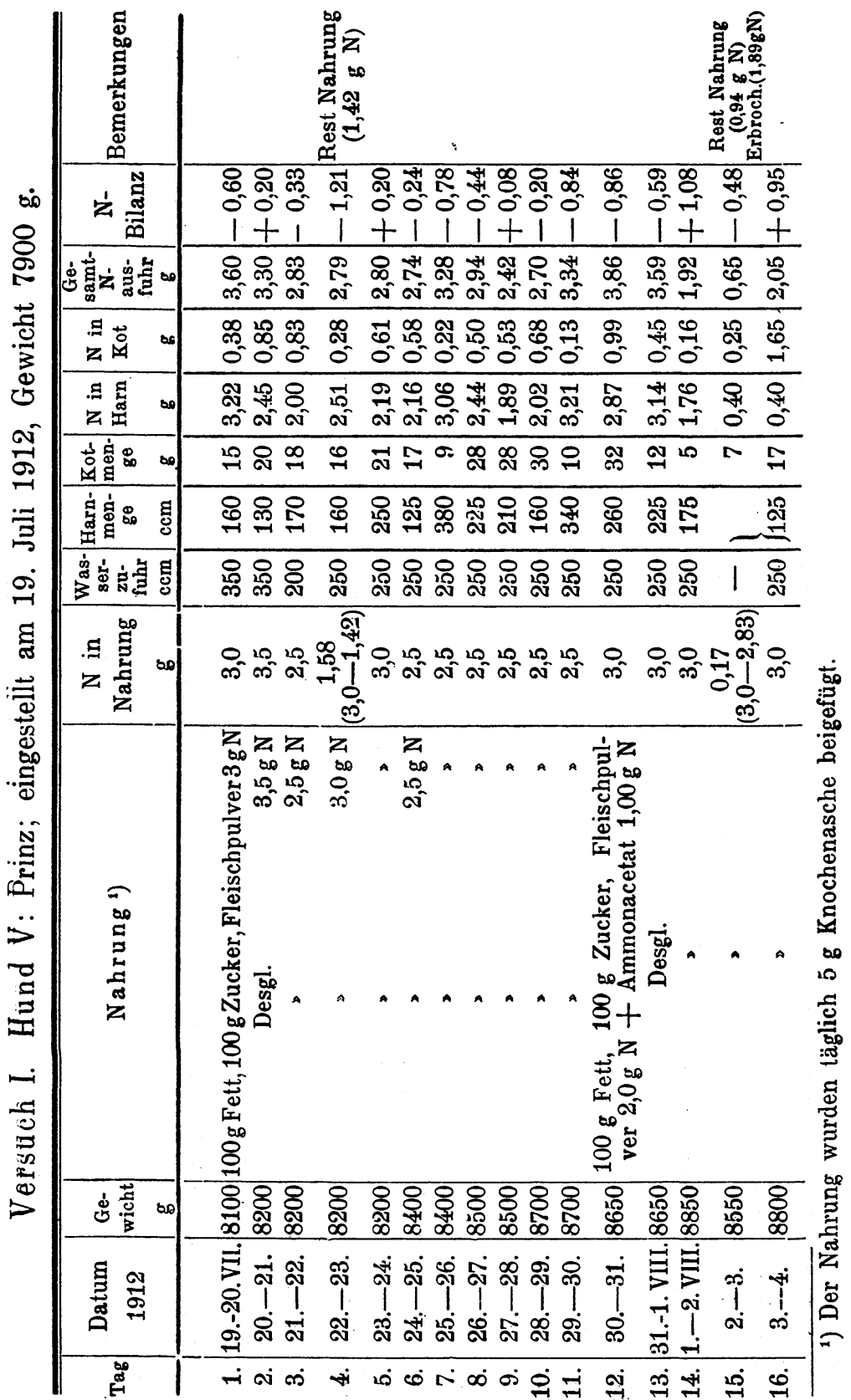




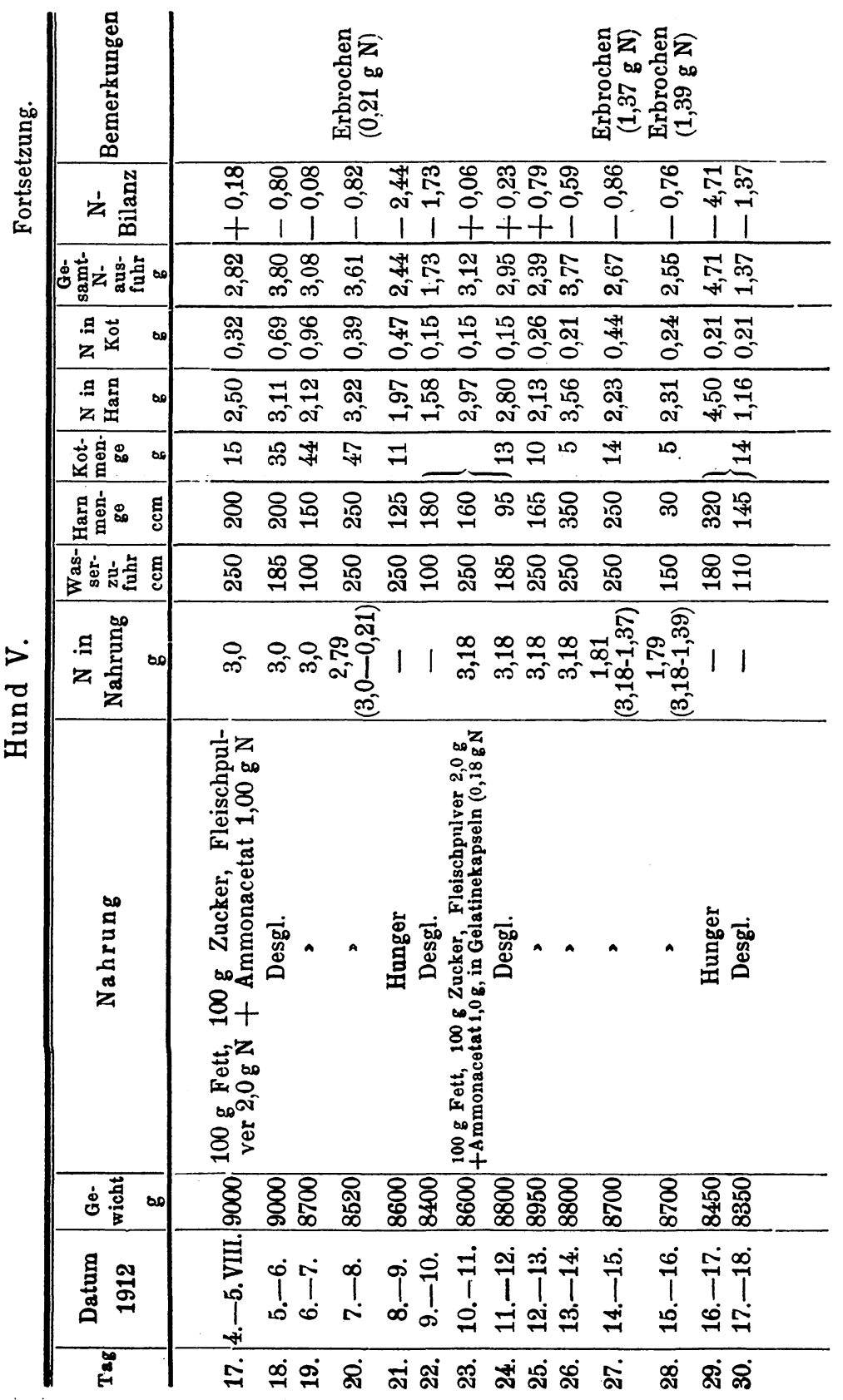


Über die synthetischen Fähigkeiten der tierischen Zelle.

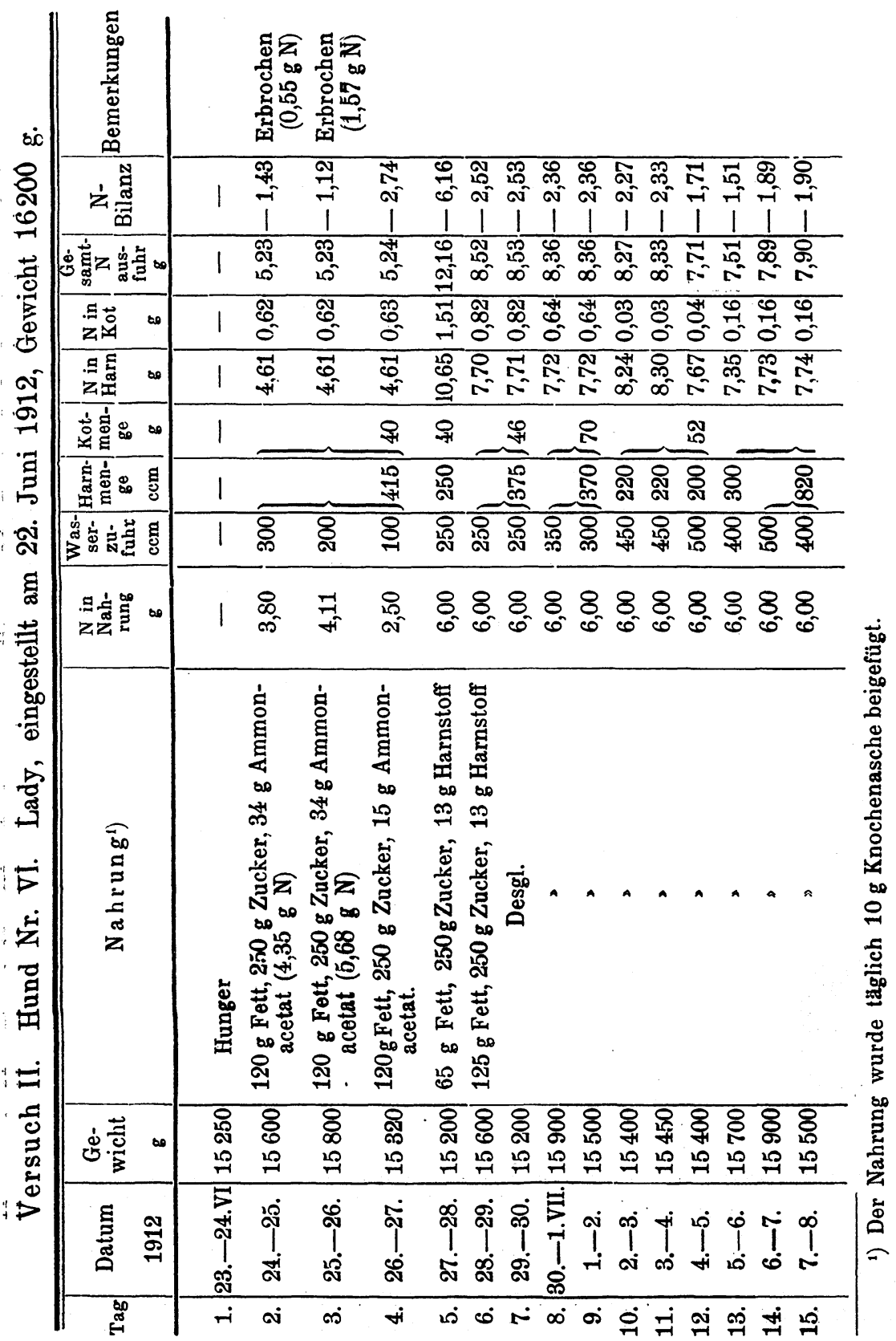


Emil Abderhalden und Paul Hirsch,

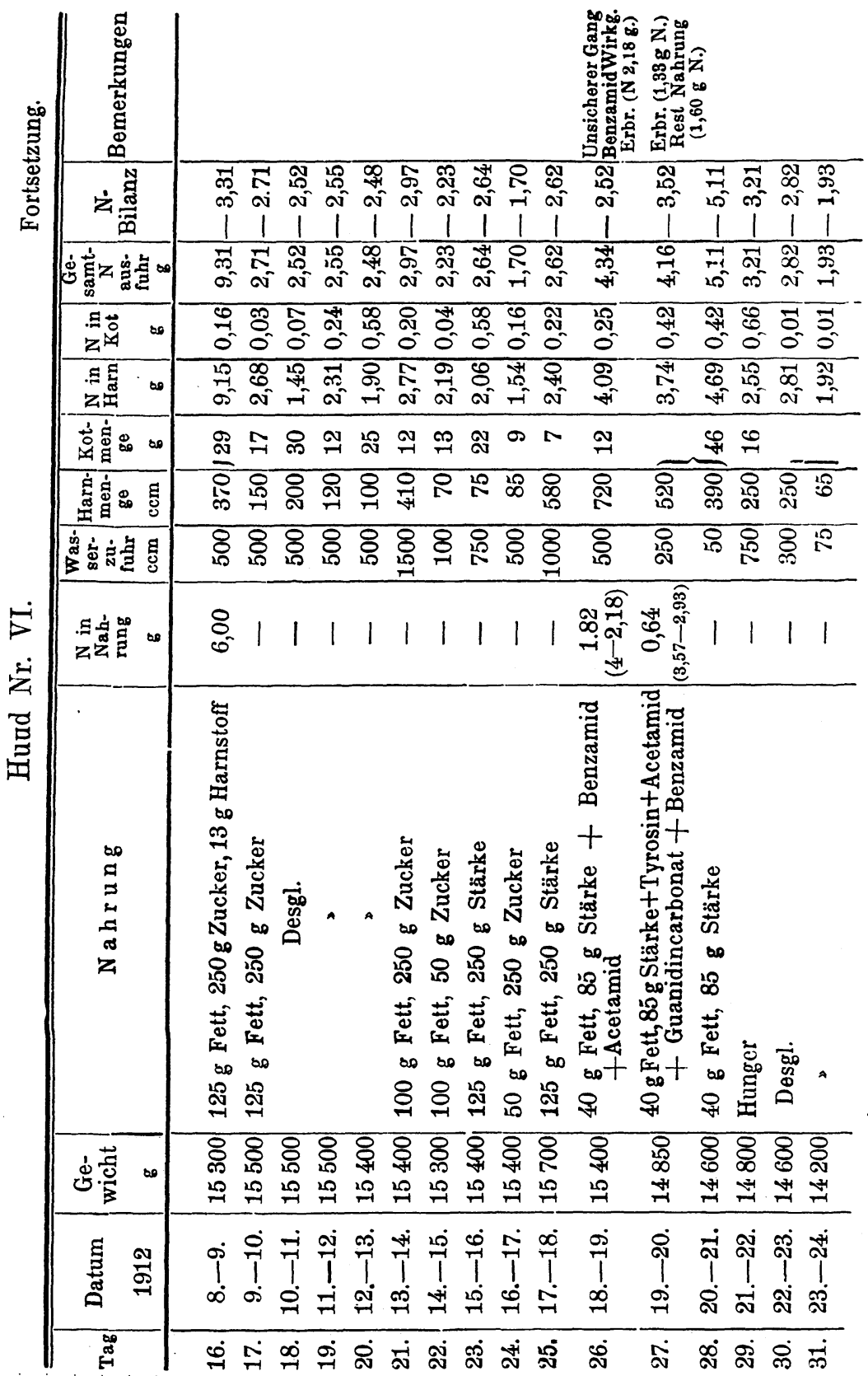




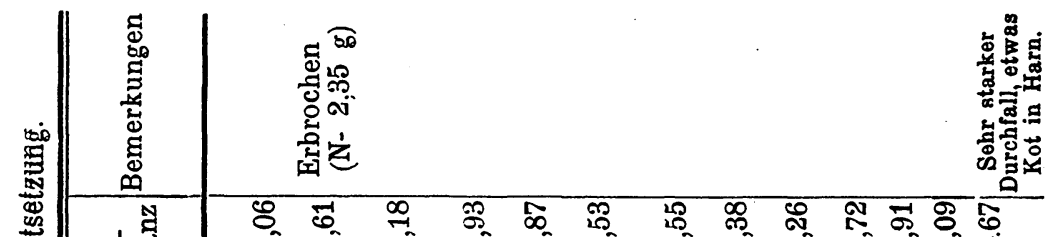

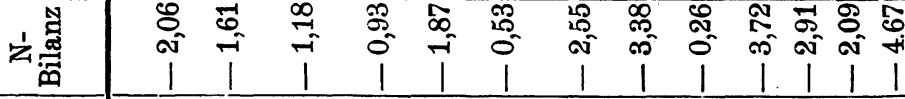
d 至

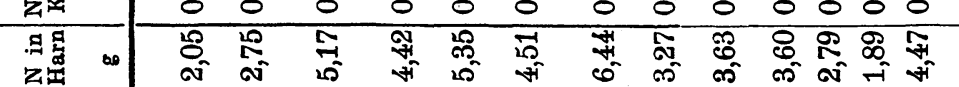

造范

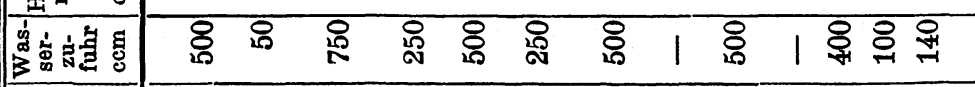

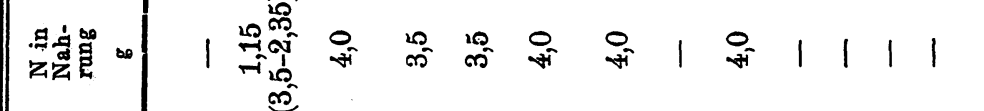
- $z_{00}^{z} z^{z}$ bo $\quad z^{z}$

\begin{tabular}{|c|c|c|c|c|c|c|c|c|c|c|}
\hline 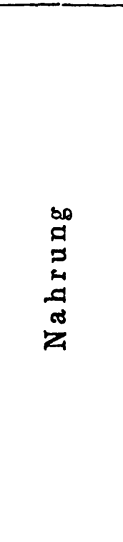 & 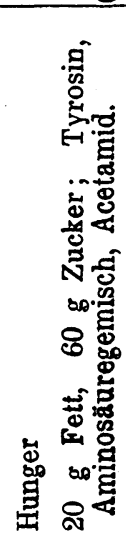 & 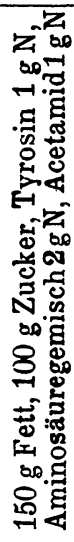 & 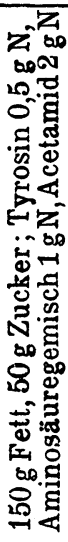 & $\begin{array}{l} \\
\dot{00} \\
\stackrel{0}{0}\end{array}$ & 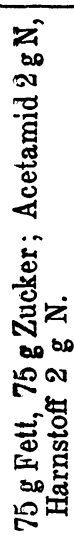 & 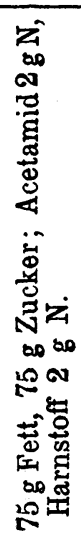 & 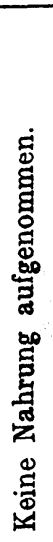 & 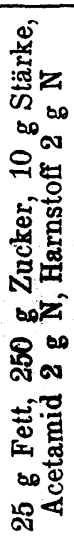 & 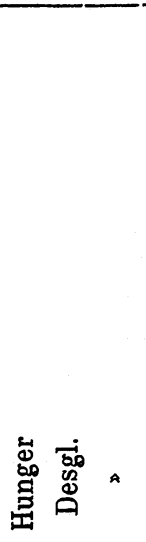 & A \\
\hline 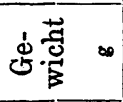 & 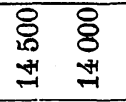 & 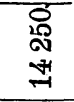 & 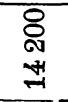 & $\begin{array}{l}8 \\
\text { क } \\
\pm \\
-1\end{array}$ & $\begin{array}{l}8 \\
8 \\
~ \\
7\end{array}$ & $\begin{array}{l}8 \\
\\
7 \\
7\end{array}$ & $\begin{array}{l}0 \\
\stackrel{0}{1} \\
\text { m } \\
\rightarrow\end{array}$ & $\begin{array}{l}8 \\
8 \\
m \\
\rightarrow 1\end{array}$ & 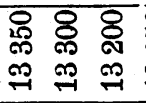 & לై \\
\hline 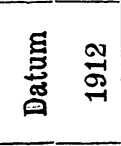 & ஸి & 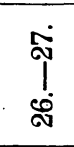 & $\underbrace{\infty}_{\text {ஸे }}$ & 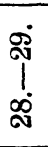 & ஷి & pi & 点 & $\stackrel{9}{i}$ & $\left.\left.\prod_{\sigma i}^{\infty}\right|_{\infty} ^{\infty}\right|_{+\infty} ^{\infty}$ & \\
\hline$\stackrel{\leftrightarrow}{\stackrel{3}{3}}$ & ชู่ & लें & חె & $\infty$ & 悉 & $\infty$ & శ్ & $\dot{\$}$ & 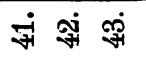 & \\
\hline
\end{tabular}




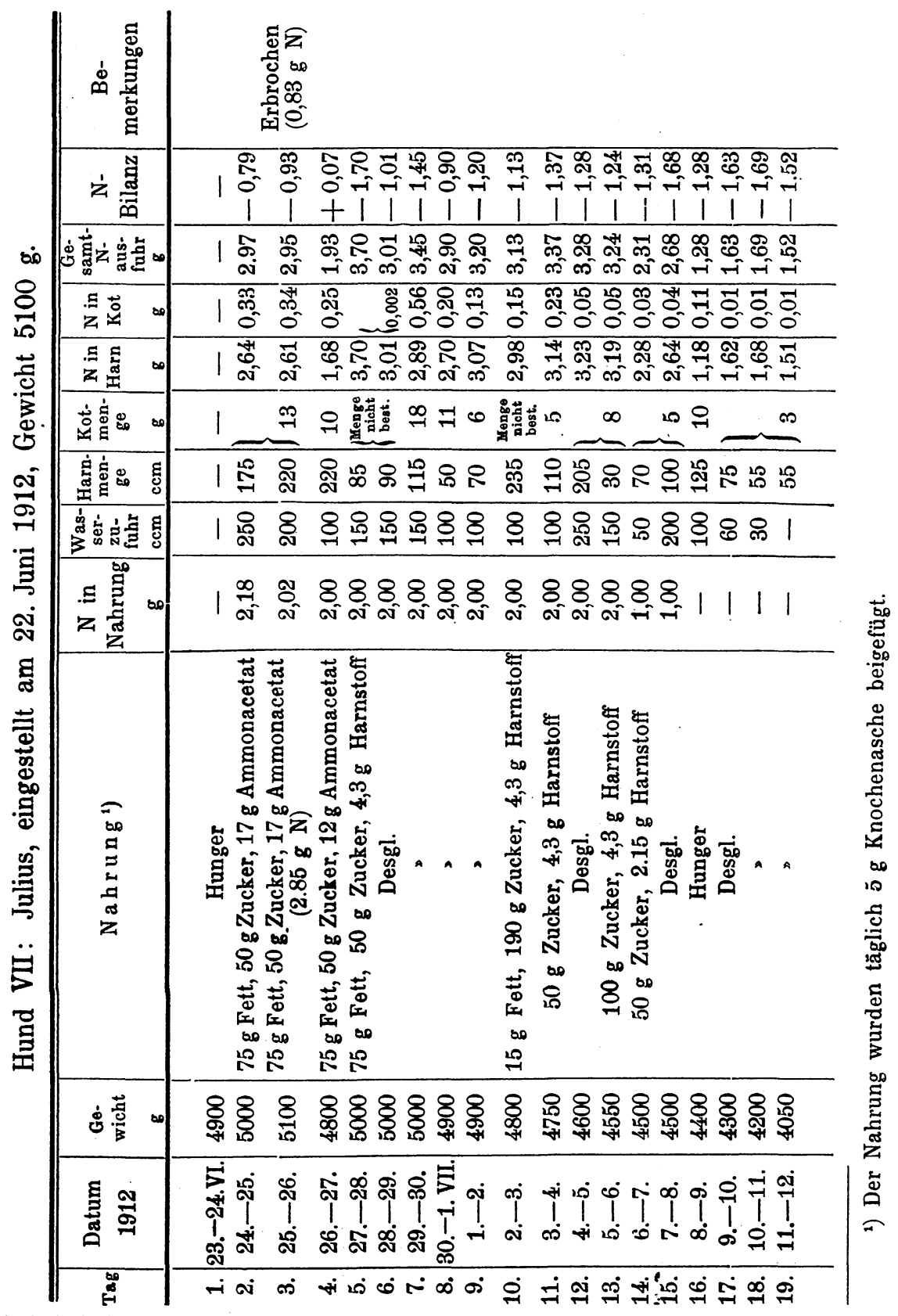


Über die synthetischen Fähigkeiten der tierischen Zelle.

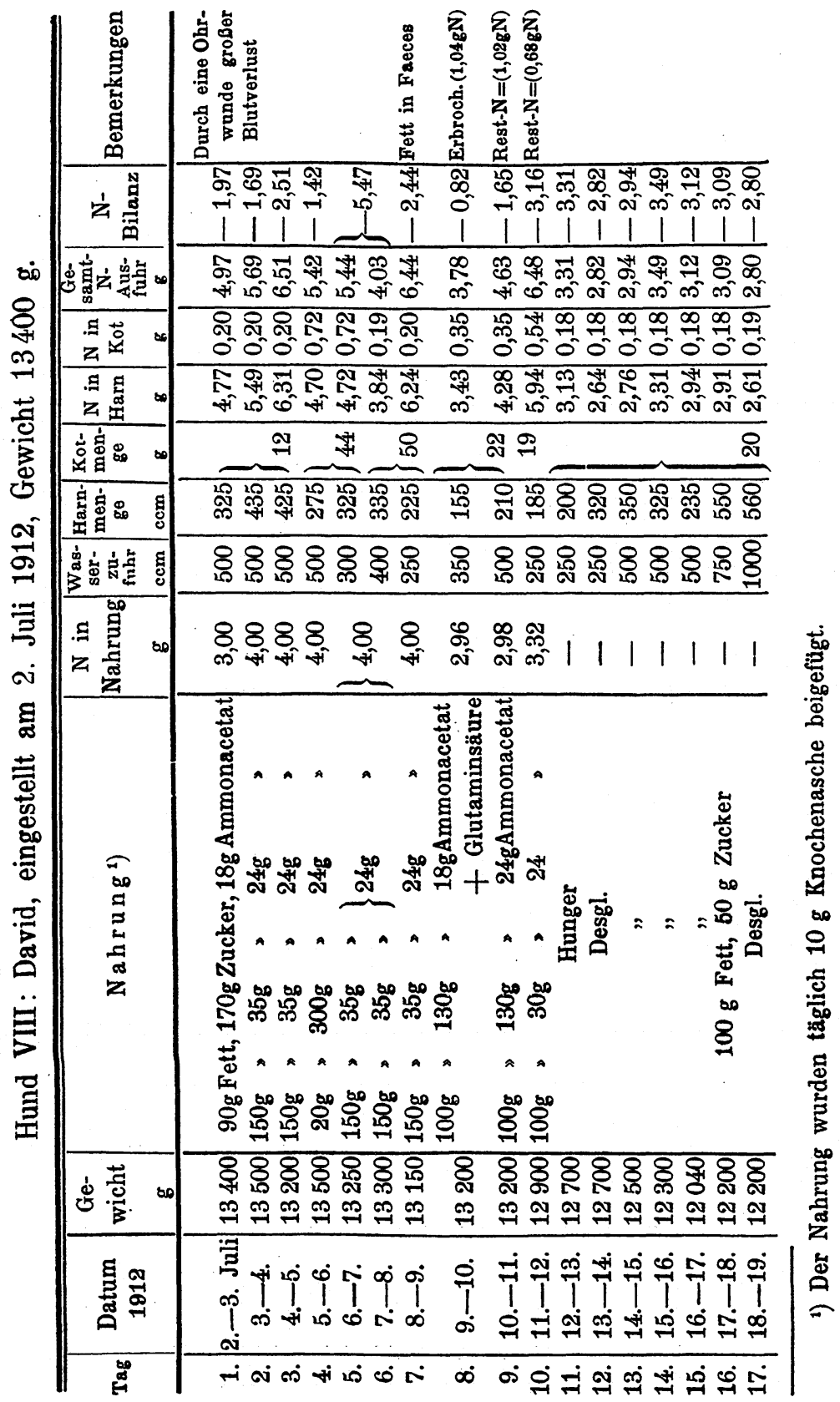




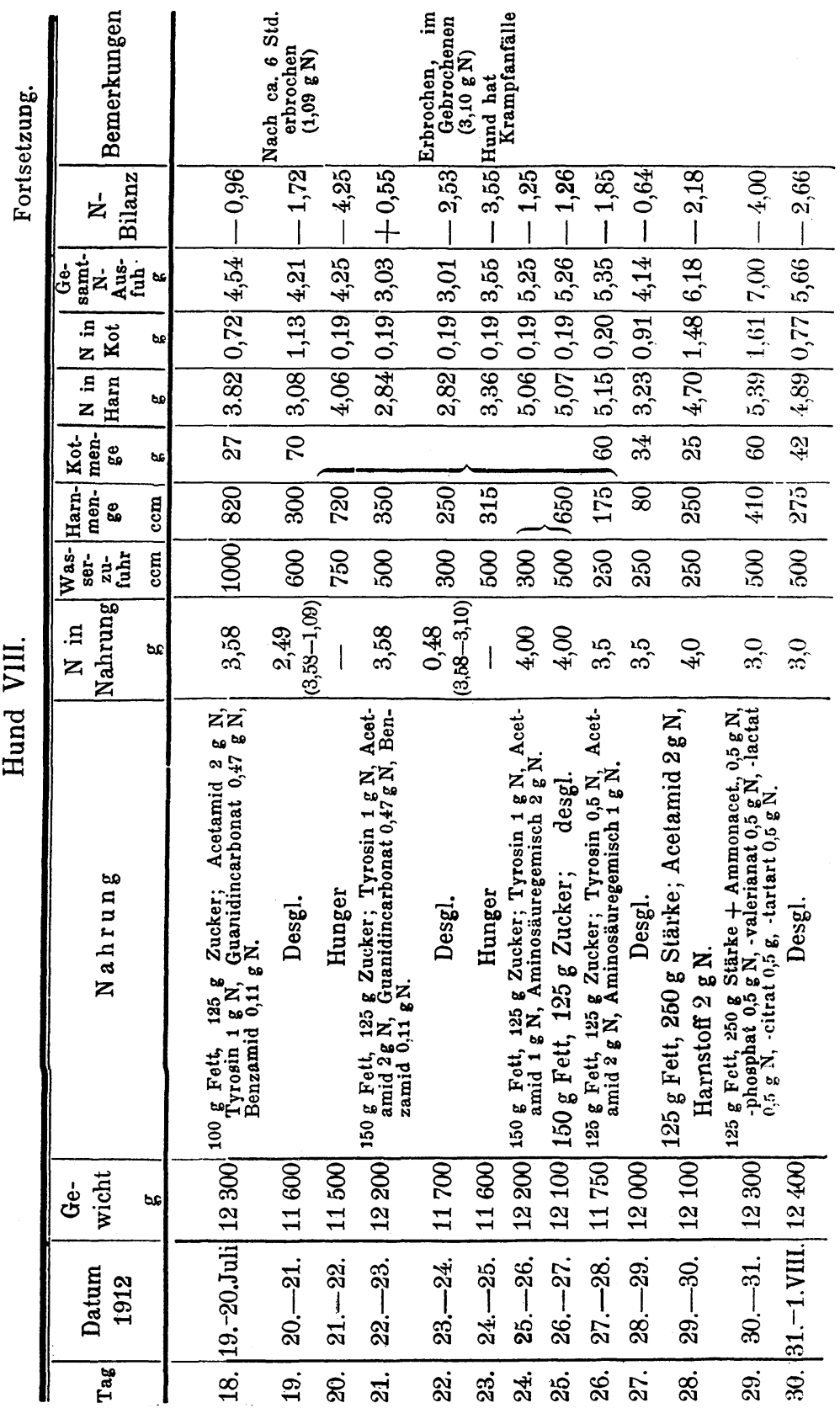


Über die synthetischen Fähigkeiten der tierischen Zelle.

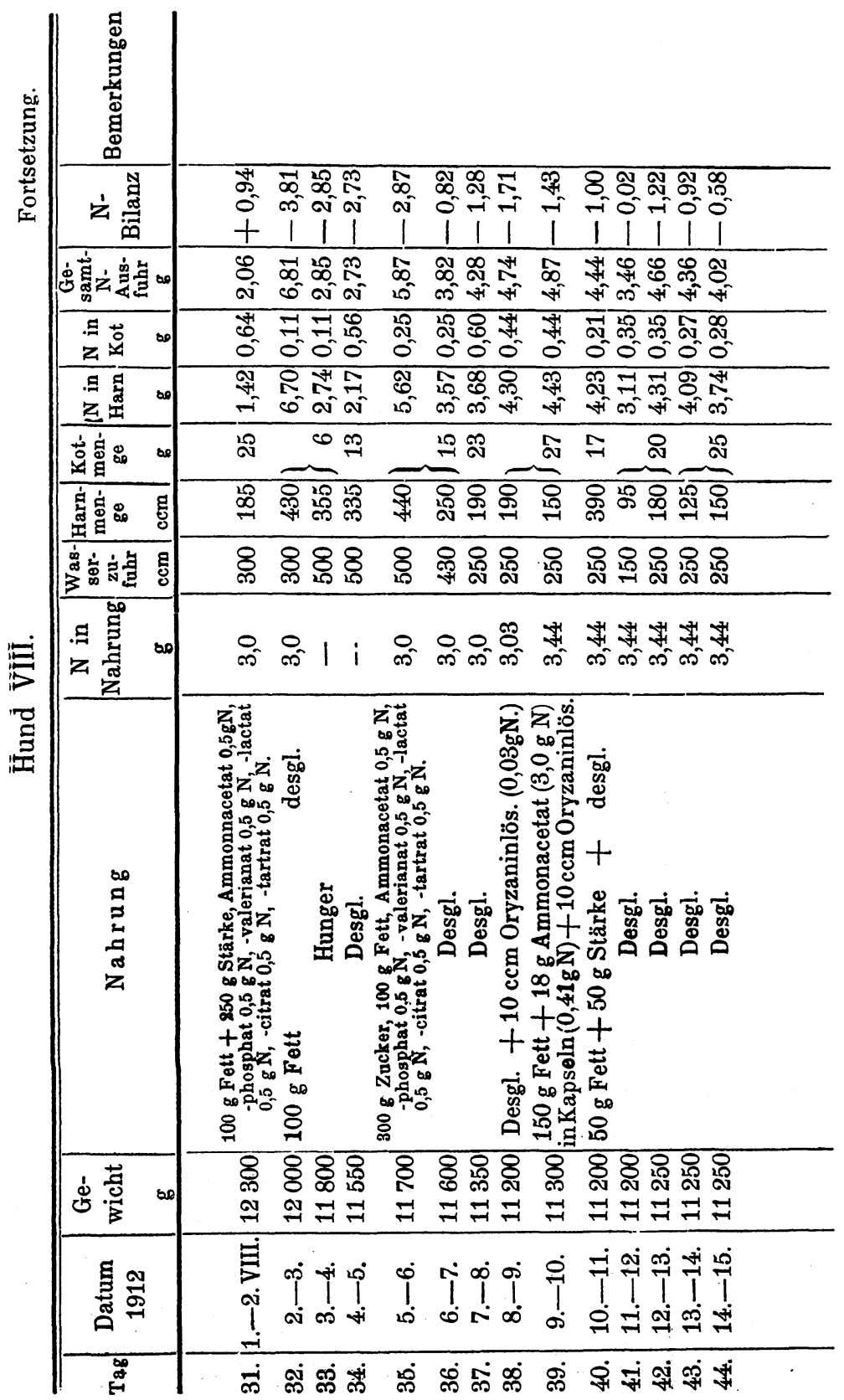


Emil Abderhalden und Paul Hirsch,

\begin{tabular}{|c|c|}
\hline 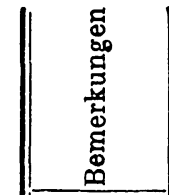 & 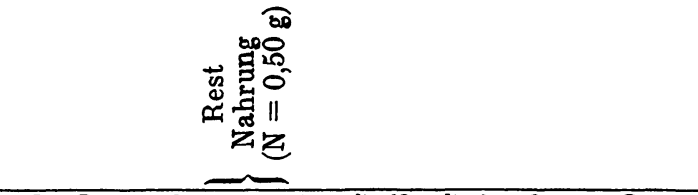 \\
\hline 玄营 & 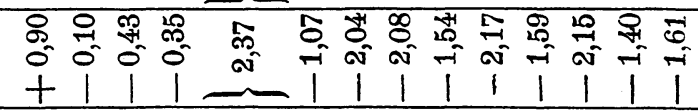 \\
\hline 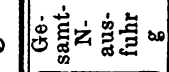 & 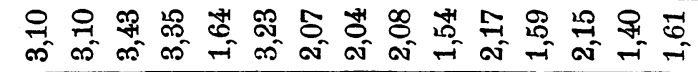 \\
\hline 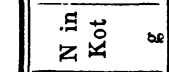 & 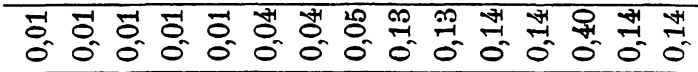 \\
\hline 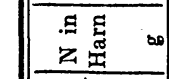 & 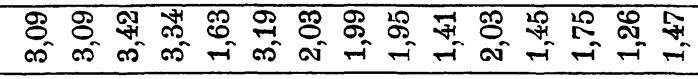 \\
\hline 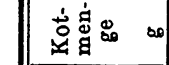 & 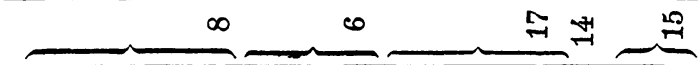 \\
\hline 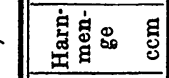 & ـ \\
\hline 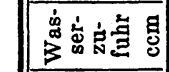 & 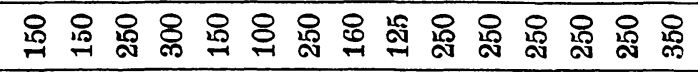 \\
\hline 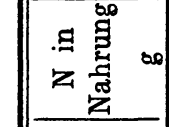 & 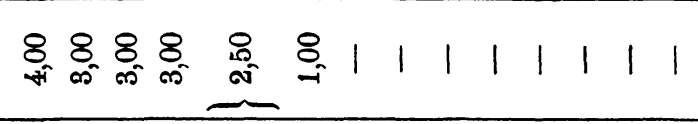 \\
\hline 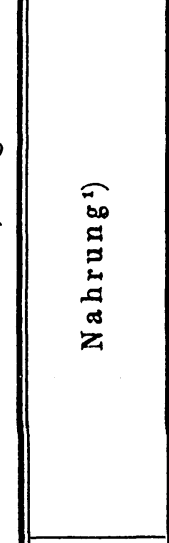 & 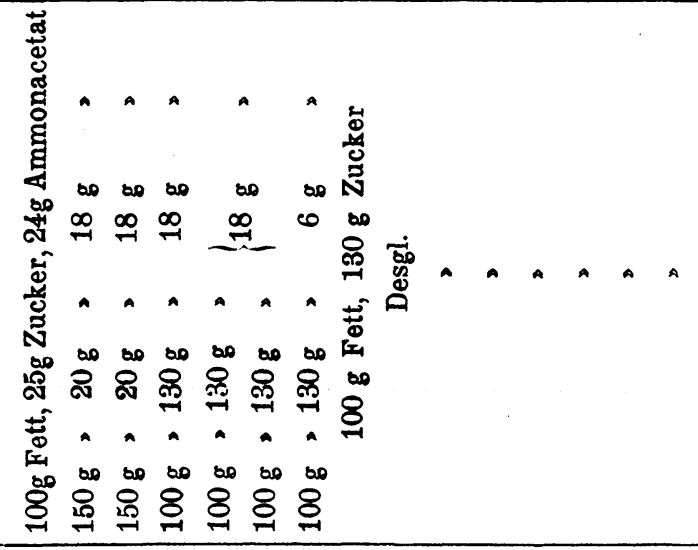 \\
\hline 造 & 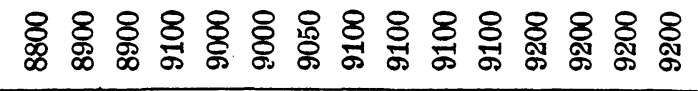 \\
\hline 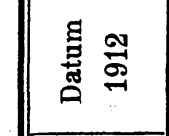 & 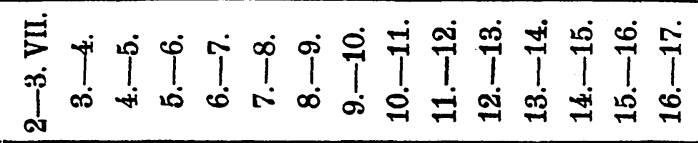 \\
\hline & 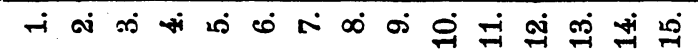 \\
\hline
\end{tabular}


Über die synthetischen Fähigkeiten der tierischen Zelle.

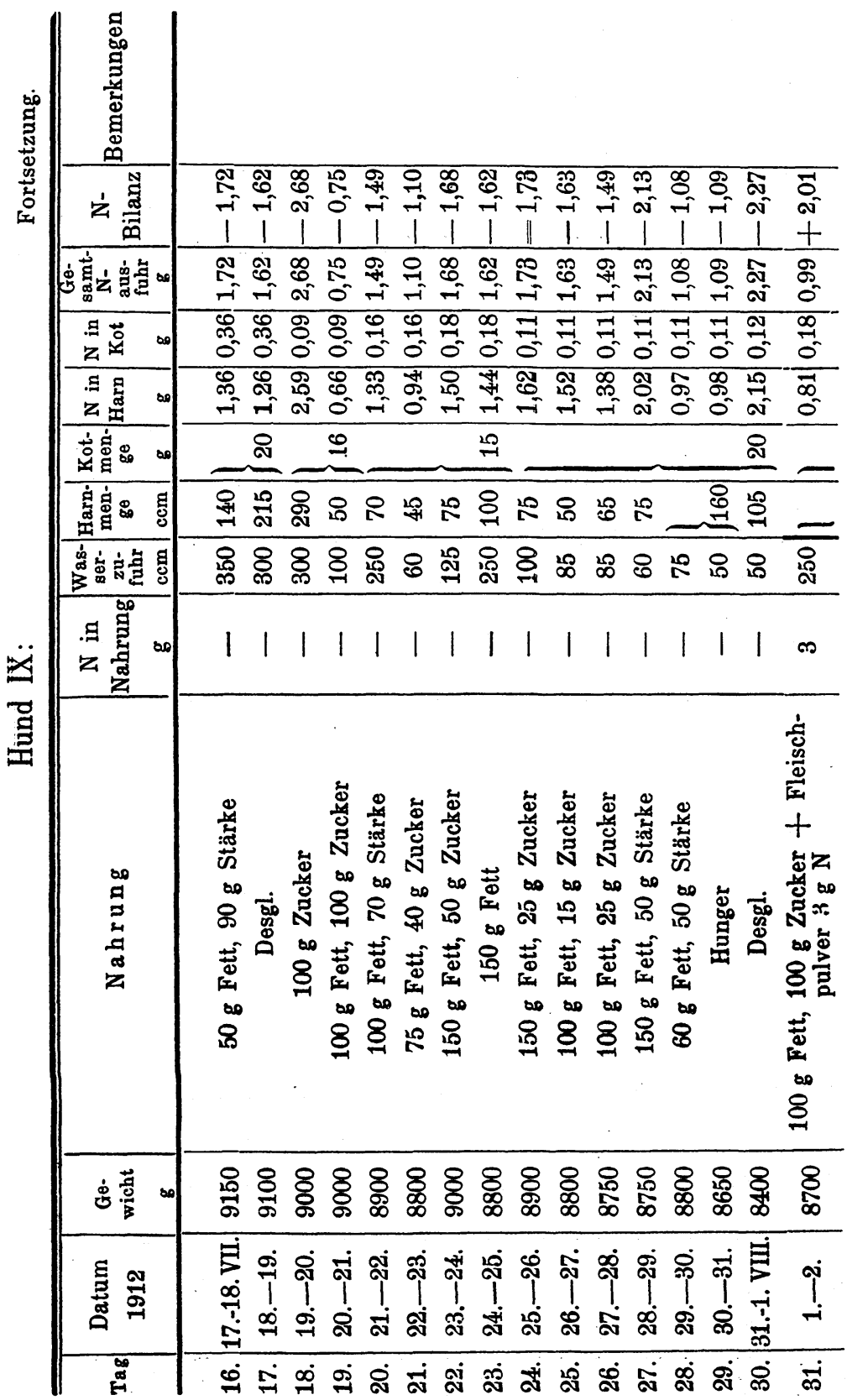




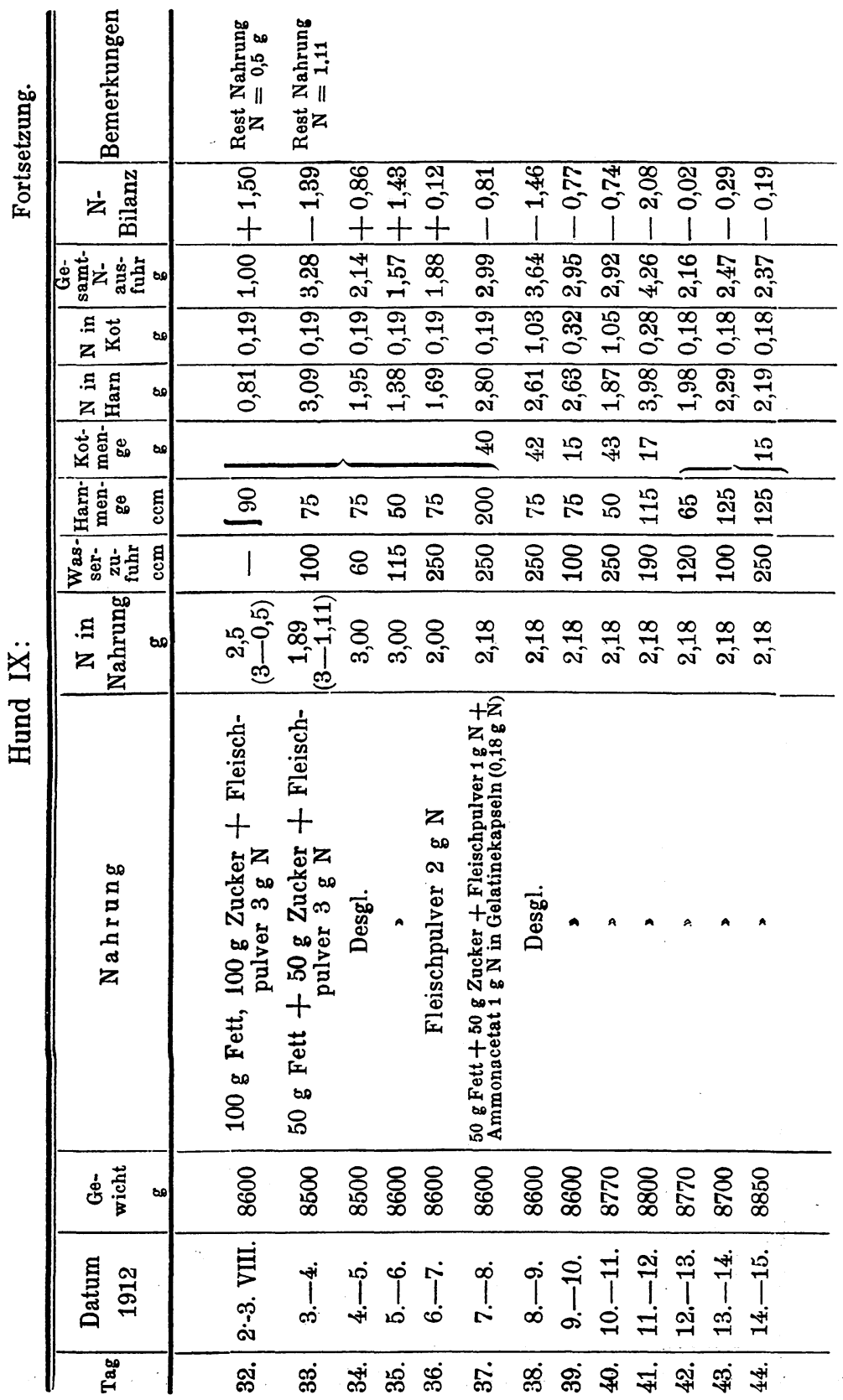


Über die synthetischen Fähigkeiten der tierischen Zelle.

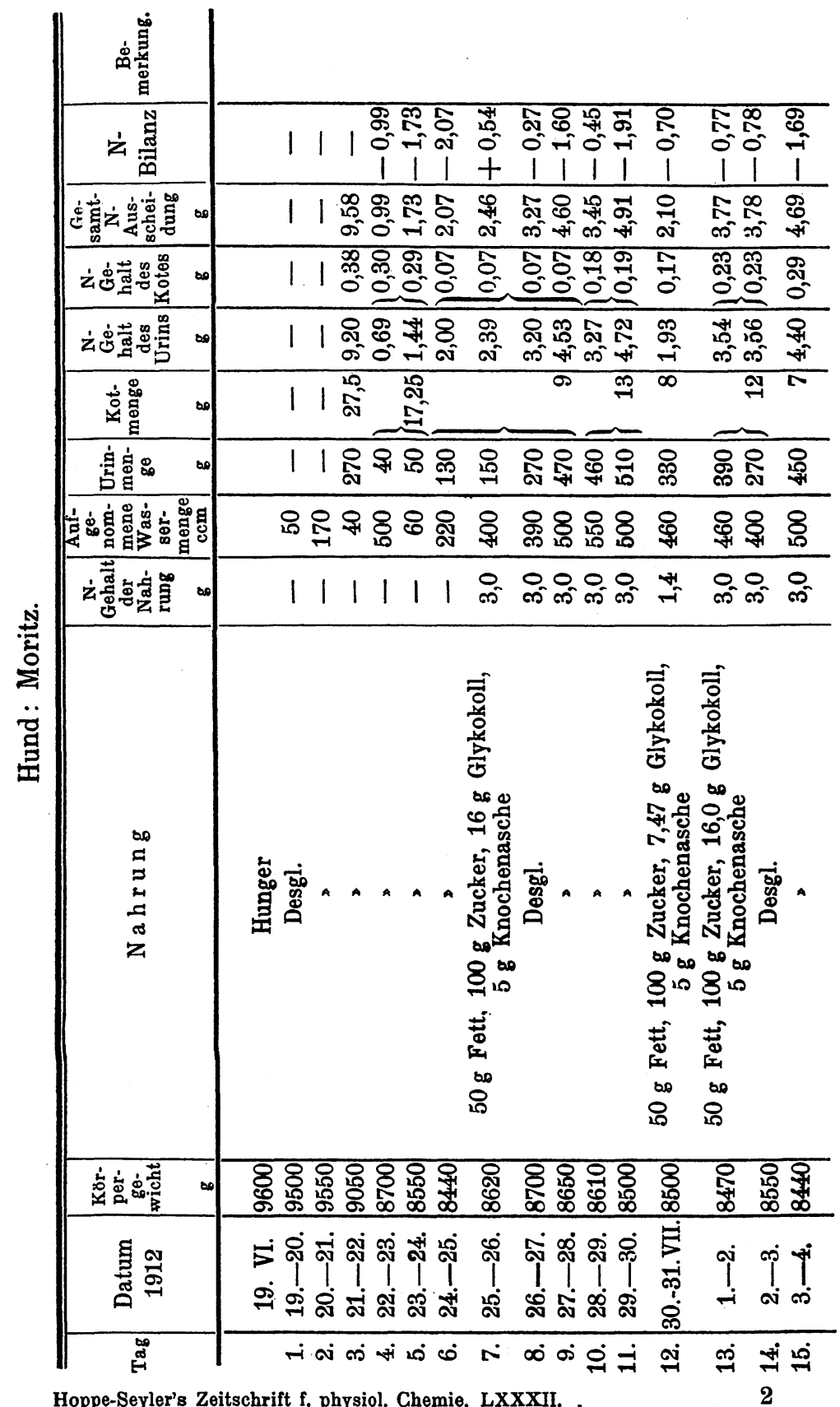

Hoppe-Seyler's Zeitschrift f. physiol. Chemie. LXXXII. . 


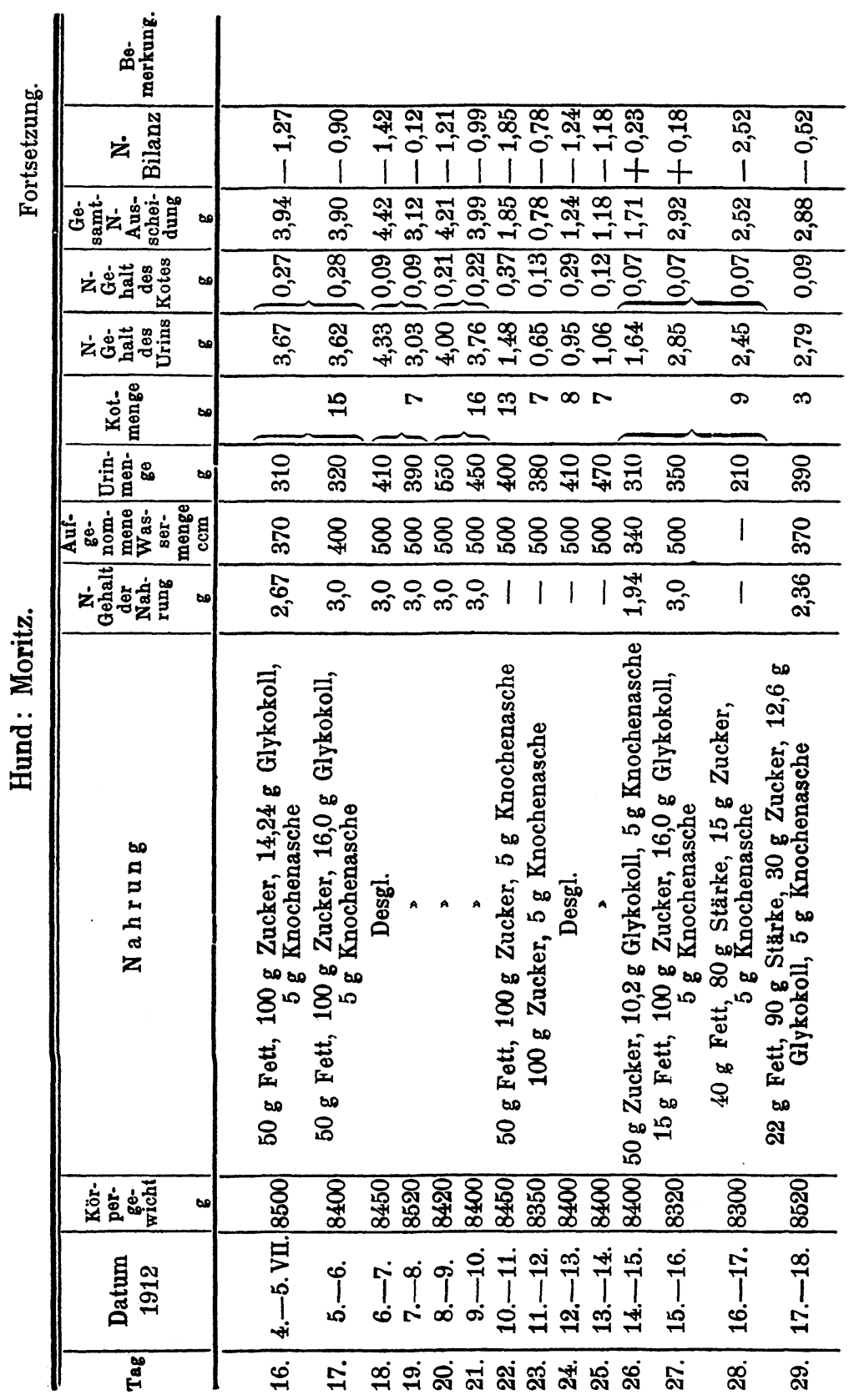


Uber die synthetischen Fähigkeiten der tierischen Zelle.

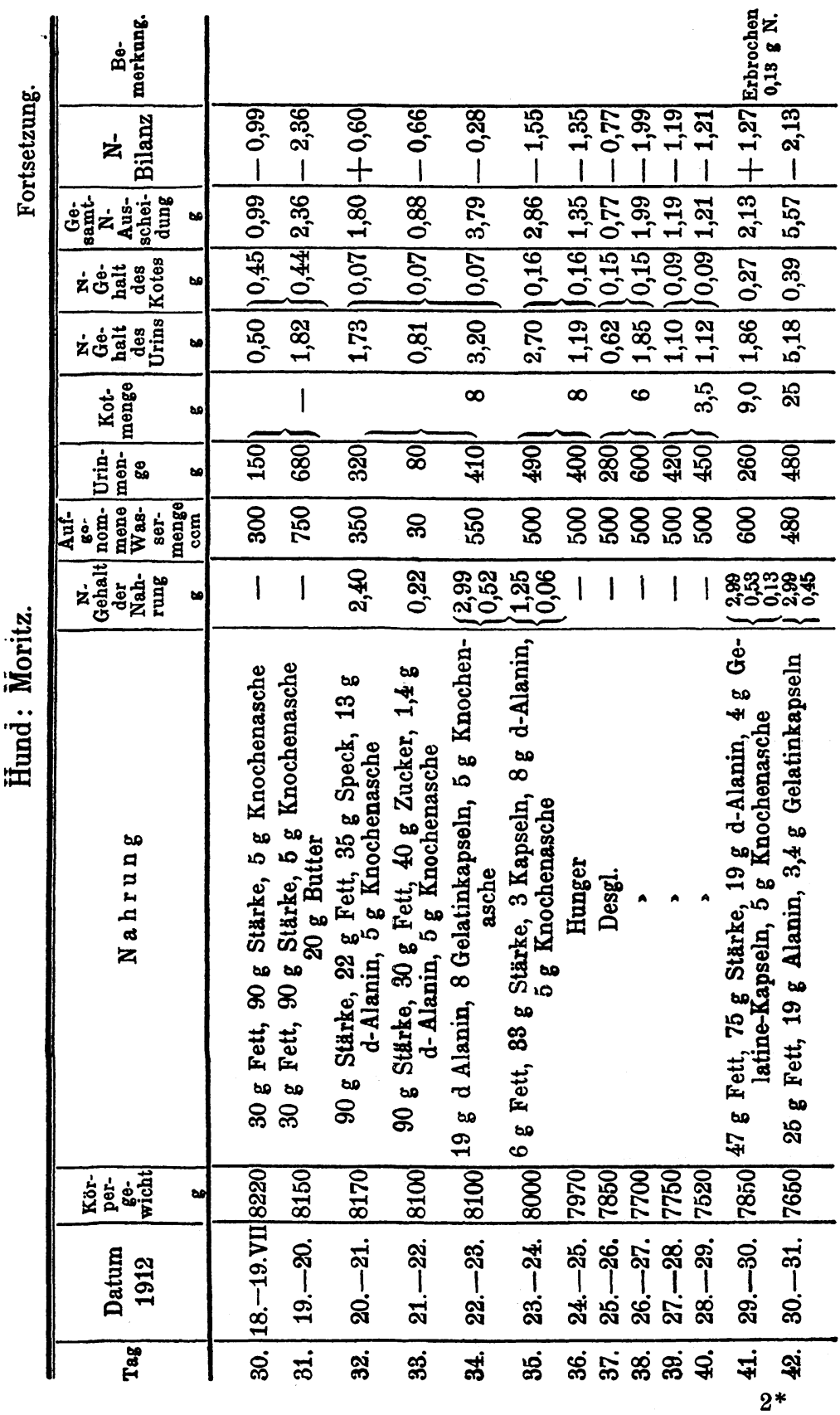


bilanz günstig $\mathrm{zu}$ beeinflussen. Der folgende Versuch zeigt, daß das nicht der Fall war. Die Versuche werden fortgesetzt. Wir ziehen aus unseren Versuchen den Schluß, daß Ammonsalze und speziell das Ammonacetat bisweilen die Stickstoffbilanz im Sinne einer Retention beeinflussen können. Irgend eine Andeutung für die Annahme, daß die tierische Zelle aus Ammoniak und stickstoffreien Verbindungen (Kohlenhydraten etc.)) Eiweiß bilden kann, ist aus unseren Ergebnissen nicht zu entnehmen. 